\title{
Hour Squared Times Nanogram per Milliliter
}

National Cancer Institute

\section{Source}

National Cancer Institute. Hour Squared Times Nanogram per Milliliter. NCI Thesaurus.

Code C85608.

Hours squared times nanograms per milliliter. 